\title{
The Unyvero Hospital-Acquired pneumonia panel for diagnosis of secondary bacterial pneumonia in COVID-19 patients
}

\author{
Chaitanya Tellapragada ${ }^{1} \cdot$ Christian G. Giske ${ }^{1,2}$ \\ Received: 11 November 2020 / Accepted: 9 February 2021 / Published online: 4 March 2021 \\ (C) The Author(s) 2021, corrected publication 2021
}

\begin{abstract}
The study was undertaken to evaluate the performance of Unyvero Hospitalized Pneumonia (HPN) panel application, a multiplex PCR-based method for the detection of bacterial pathogens from lower respiratory tract (LRT) samples, obtained from COVID19 patients with suspected secondary hospital-acquired pneumonia. Residual LRT samples obtained from critically ill COVID19 patients with predetermined microbiological culture results were tested using the Unyvero HPN Application. Performance evaluation of the HPN Application was carried out using the standard-of-care (SoC) microbiological culture findings as the reference method. Eighty-three LRT samples were used in the evaluation. The HPN Application had a full concordance with SoC findings in 59/83 (71\%) samples. The new method detected additional bacterial species in $21(25 \%)$ and failed at detecting a bacterial species present in lower respiratory culture in $3(3.6 \%)$ samples. Overall the sensitivity, specificity, positive, and negative predictive values of the HPN Application were 95.1\% (95\%CI 96.5-98.3\%), 98.3\% (95\% CI 97.5-98.9\%), 71.6\% (95\% CI 61.0-80.3\%), and 99.8\% (95\% CI 99.3-99.9\%), respectively. In conclusion, the HPN Application demonstrated higher diagnostic yield in comparison with the culture and generated results within $5 \mathrm{~h}$.
\end{abstract}

Keywords Hospital-Acquired pneumonia $\cdot$ COVID-19 $\cdot$ Rapid-diagnostic tests $\cdot$ Bacterial pneumonia $\cdot$ Multiplex-PCR

\section{Introduction}

Lower respiratory tract infections (LRTIs) have a diverse microbial etiology including bacterial, viral, and fungal pathogens. Early and accurate identification of the infectious etiology causing LRTIs is crucial for deciding the course of treatment with reference to selection of appropriate antimicrobial therapy. Pathogen-specific antimicrobial therapy among patients with more severe form of LRTIs, such as communityand ventilator-associated pneumonia, has been reported to reduce the length of hospital-stay, health care costs, and adverse clinical outcomes [1]. Historically, etiological diagnosis of bacterial pneumonia has primarily been based on the microbiological culture findings. Minimum turn-around time for

Chaitanya Tellapragada

Chaitanya.tellapragada@ki.se

1 Divison of Clinical Microbiology, Department of Laboratory Medicine, Karolinska Institute, Alfred Nobels Allé 8, 14183 Stockholm, Sweden

2 Division of Clinical Microbiology, Karolinska University Hospital, Stockholm, Sweden results from the time of sample inoculation for culture and antimicrobial susceptibility testing is approximately $36-48$ h. Sensitivity of the microbiological culture techniques for recovery of bacterial pathogens from lower respiratory tract samples can be hindered by several factors including (i) prior administration of antibiotics, (ii) poor quality and low quantity of the sample, (iii) overgrowth of commensal respiratory tract microbiota, and (iv) technical expertise of the microbiologist reading the culture plates. Semi-quantitative cultures of respiratory samples often cannot distinguish colonizer from pathogen. This problem can be overcome by using quantitative culture techniques that are more conclusive but are more laborious and cumbersome to perform.

Amidst these pre-existing challenges with reference to etiological diagnosis of LRTIs, COVID-19 has rapidly emerged as a major public health concern, worldwide. Current estimates suggest that nearly $80 \%$ of the patients admitted in the ICU with COVID-19 receive antibiotics [2]. In contrast, from a recent meta-analysis that included 24 studies from various countries, it was reported that bacterial co-infections were reported in only $7-15 \%$ of the patients admitted in the ICU [3]. Antibiotic therapy in the absence of etiological diagnosis of infection has 
both clinical and public health implications. Inappropriate use of antibiotics is a well-established driver for emergence of antimicrobial resistance among bacterial pathogens. Given this context, it is important to verify whether newer diagnostic modalities capable of detecting bacterial pathogens from native clinical specimens can be useful in providing early and accurate etiological diagnosis of pneumonia among critically ill COVID-19 patients [4].

The Unyvero Hospitalized Pneumonia (HPN) Application (Curetis GmbH, Germany) is a commercially available CE-IVD rapid multiplex polymerase chain reaction (PCR)-based diagnostic system for use on lower respiratory tract specimens. A closed cartridge-based approach is used for specimen lysis, DNA extraction, PCR, and array hybridization; turnaround time is approximately four and a half hours. The panel detects the most common species observed in patients with hospitalacquired and ventilator-associated pneumonia, in addition to Mycoplasma pneumoniae, Chlamydia pneumoniae, Legionella pneumophila, and Pneumocystis jirovecii; it also detects seventeen antibiotic resistance markers (Table 1). Herein, we evaluated the performance of the Unyvero HPN Application, in comparison with standardof care microbiological culture findings, for detection of bacterial pathogens from lower respiratory tract (LRT) samples obtained from critically ill COVID-19 patients.

\section{Material and methods}

\section{Study details}

A laboratory-based comparative study was undertaken at the Department of Clinical Microbiology, Karolinska University Hospital, Stockholm, Sweden, from April 2020 through June 2020, during the first wave of the COVID-19 pandemic in Stockholm, Sweden.

\section{Study samples}

Multiple aliquots of remnant lower respiratory samples were frozen at $-80^{\circ} \mathrm{C}$ within $24 \mathrm{~h}$ of collection, and they were kept frozen at $-80{ }^{\circ} \mathrm{C}$ in the biobank of Karolinska University Laboratory and thawed at room temperature immediately prior to testing in the present study. The biobank consisted of residual LRT samples (after standard cultures) received at the clinical microbiology laboratory during March 2020-June 2020, obtained from hospitalized COVID-19 patients in Stockholm with a clinical suspicion of secondary bacterial infection. Microbiology culture reports, sample types, and baseline demographic characteristics of the subjects were extracted from the laboratory information system using the laboratory identification numbers of the samples available in the biobank. Selection of the samples was carried out based on the
Table 1 Unyvero HPN panel targets

\begin{tabular}{|c|c|c|}
\hline \multirow{2}{*}{$\frac{\text { Unyvero HPN panel microorganisms }}{\text { Acinetobacter baumannii complex }}$} & \multicolumn{2}{|c|}{ Unyvero HPN panel antibiotic resistance markers } \\
\hline & Carbapenems & $b l a_{\mathrm{KPC}}$ \\
\hline Chlamydia pneumoniae & & $b l a_{\mathrm{IMP}}$ \\
\hline Citrobacter freundii & & $b l a_{\mathrm{NDM}}$ \\
\hline Enterobacter cloacae complex & & $b l a_{\text {OXA-23 }}$ \\
\hline Escherichia coli & & $b l a_{\text {OXA-24/40 }}$ \\
\hline Haemophilus influenzae & & $b l a_{\mathrm{OXA}-48}$ \\
\hline Klebsiella aerogenes (E. aerogenes) & & $b l a_{\mathrm{OXA}-58}$ \\
\hline Klebsiella oxytoca & & $b l a_{\mathrm{VIM}}$ \\
\hline Klebsiella pneumoniae & 3rd-generation Cephalosporins & $b l a_{\text {СтХ-м }}$ \\
\hline Klebsiella variicola & Fluoroquinolones & gyrA83 \\
\hline Legionella pneumophila & & gyrA87 \\
\hline Moraxella catarrhalis & Macrolide/Lincosamide & ermB \\
\hline Morganella morganii & Oxacillins & mecA \\
\hline Mycoplasma pneumoniae & & $\operatorname{mec} C$ \\
\hline Pneumocystis jirovecii & Penicillins & $b l a_{\mathrm{TEM}}$ \\
\hline Proteus spp. & & $b^{b H} a_{\mathrm{SHV}}$ \\
\hline Pseudomonas aeruginosa & Sulfonamides & sull \\
\hline \multicolumn{3}{|l|}{ Serratia marcescens } \\
\hline \multicolumn{3}{|l|}{ Staphylococcus aureus } \\
\hline \multicolumn{3}{|l|}{ Stenotrophomonas maltophilia } \\
\hline Streptococcus pneumoniae & & \\
\hline
\end{tabular}


following inclusion criteria: (i) samples obtained from adult patients, (ii) obtained from subjects admitted in the intensivecare unit, and (iii) samples that were either positive for normal respiratory microbiota or positive for one or more of the organism targets of the Unyvero HPN panel. Selected samples were tested with the Unyvero HPN Application.

\section{Standard-of-Care (SoC) testing}

Quantitative culture methods were used for both invasive and non-invasive sample types at the study laboratory, and interpretation of the culture plates was done in accordance with the standard recommendations[5]. Detailed description of the sample volumes, culture media used, and incubation conditions for various bacterial species are described previously [6]. Results from the cultures were reported in colony-forming units (CFU) per milliliter. Identification of the bacterial species was performed using MALDI-TOF (Bruker), as per the manufacturer's instructions. Antimicrobial susceptibility testing was performed using the disk-diffusion test, and result interpretation was according to the EUCAST guidelines, version 8.0, Jan 2020 (www.eucast.org.). Detection of atypical agents such as Mycoplasma pneumoniae, Chlamydophila pneumoniae, and Legionella pneumophila was performed at the study laboratory only on samples, when indicated by the clinician. For those samples, the DNA was extracted and subjected to a multiplex real-time PCR (Allplex Respiratory Panel-4, Seegene Inc.) as per the manufacturer's instructions. Testing for Pneumocystis jirovecii was not indicated by the clinicians for any of the present study samples and hence not performed.

\section{Testing of the study samples using Unyvero HPN Application}

Frozen samples were thawed for testing using the Unyvero HPN Application. The Unyvero HPN panel is a multiplexed molecular rapid diagnostic test, specifically designed for the detection of lower respiratory tract infections. The Unyvero analysis was performed on the Unyvero System, consisting of a Lysator, Analyzer, and Cockpit, and clinical specimens were processed in accordance with manufacturer's (Curetis $\mathrm{GmbH}$, Holzgerlingen, Germany) instructions for use and site-specific laboratory protocols to ensure safe handling of specimens and maintenance of quality. Briefly, $180 \mu \mathrm{L}$ of the respiratory sample was added (in a biosafety cabinet) to the provided sample tube containing lysis buffer and loaded in the lysator for a 30-min lysis and then transferred to the provided cartridge and loaded in the analyzer for a 4.5 -h PCR and detection. List of the organisms and the antimicrobial resistance genes available on the Unyvero HPN panel for testing are shown in Table 1. The results were qualitatively reported as positive or negative for each organism/resistance marker.

\section{Comparison of results from HPN Application results with SoC}

Concordance of the HPN Application with SoC was considered when (i) same panel organism(s) detected by both HPN Application and SoC, (ii) no panel organisms detected by both HPN Application and SoC, and (iii) no organism detected by HPN Application, culture positive for non-panel organism. Discordant results were considered when (i) the same sample positive for different panel organisms by HPN Application and SoC; (ii) culture positive for HPN panel organism, HPN Application negative; and (iii) HPN Application positive, culture-negative for the same organism. A sample was defined as both concordant and discordant if the HPN panel, and SoC results had the same organism plus an additional panel organism reported by either assay with were positive according the HPN panel cutoffs. Please refer to list of concordant and discordant samples in the supplementary section (Tables S3 S5).

\section{Statistical analysis}

Pathogens detected by HPN were compared to SoC results to determine overall clinical sensitivity, specificity, positive, and negative predictive values (PPV and NPV, respectively) for all pathogens combined, together with confidence intervals according to the Wilson score method [7]. Sensitivity was calculated by \# true positives/(\# true positives + \# false negatives), specificity was calculated by \# true negatives /(\# true negatives + \# false positives), PPV was calculated by \# true positives/(\# true positives + \# false positives), and NPV was calculated by \# true negatives/(\# true negatives + \# false negatives).

\section{Results}

\section{Sample characteristics}

In total, we tested 83 samples consisting of $61(73.5 \%)$ tracheal secretions, 11 (13.4\%) bronchoalveolar lavage (BAL), 8 $(9.7 \%)$ protected specimen brush (PSB), 2 (2.4\%) bronchial secretions, and $1(1.2 \%)$ sputum sample. The 83 samples were obtained from 68 subjects (one sample from 57 unique subjects, two samples each from 7 subjects, and three samples each from 4 subjects). The multiple samples from same patient were taken on different sampling days in most cases, as indicated by the ordering clinician (Table S1). Seventy-four percent (50/68) of the study subjects were male and $26 \%(18 / 68)$ were female. The mean age of the study subjects was 58.8 \pm 11.3 years. 


\section{Microbial etiology of the study samples determined using SoC}

Of the total 83 samples, one bacterial species was isolated from $40(48 \%)$ and two bacterial species were isolated from $18(22 \%)$ samples. Normal respiratory microbiota was isolated in $25(30 \%)$ samples. The most commonly isolated organisms were $S$. aureus $(23 / 83 ; 27.7 \%)$ followed by $E$. coli and $K$. aerogenes in $5(6.0 \%)$ samples each (Table 2). When compared against the list of organisms, available on the HPN panel, the present sample cohort comprised of 37 (44.6\%) samples with one organism, $12(14.5 \%)$ samples with two organisms, and $34(41.0 \%)$ samples with no organisms. Eleven (13.3\%) samples were positive with 11 non-panel organisms (Table S2).

\section{Microbial etiology of the study samples determined using HPN Application}

Of the 83 samples tested, one bacterial species was detected from 34 (41.4\%), two bacterial species among 19 (23.1\%), and three bacterial species were detected among $3(3.6 \%)$ samples. No bacteria were detected from $27(32.5 \%)$ samples. The most detected species were $S$. aureus $(31 / 83 ; 37.4 \%)$ followed by $P$. aeruginosa $(9 / 83 ; 10.9 \%)$ and $S$. marcescens $(8 / 83$; 9.6\%) samples (Table 2).

\section{Comparison of results from HPN Application with SoC testing}

When comparing results between HPN and SoC for individual panel analytes, sensitivity, specificity, positive predictive value, and negative predictive values of the HPN application in comparison with SoC culture were 95.1\% (95\%CI 86.5-98.3\%), 98.3\% (95\%CI 97.5-98.9\%), $71.6 \%$ (95\%CI 61.0-80.3\%), and 99.8\% (95\%CI $99.3-$ $99.9 \%)$, respectively (Table 2$)$. In three cases $(1 \times$ S. aureus, $1 \times$ Proteus spp., $1 \times K$. pneumoniae), SoC reported a pathogen concentration below the recommended reporting threshold for BAL specimens of $10^{3} \mathrm{CFU} / \mathrm{mL}$ (10). Such cases were considered "subclinical" (i.e., reporting does no merit patient treatment) and were regarded as a $\mathrm{SoC}$ negative result for this study. Another sample (K. aerogenes) which was not quantitated by SoC was therefore also regarded as $\mathrm{SoC}$ negative. Overall comparison of the results for concordance between both methods is shown below (Table 3), and sample-wise comparison of the organism yield between both methods is listed below (Table 4).
Table 2 Concordance between Unyvero HPN panel and SoC results by organism

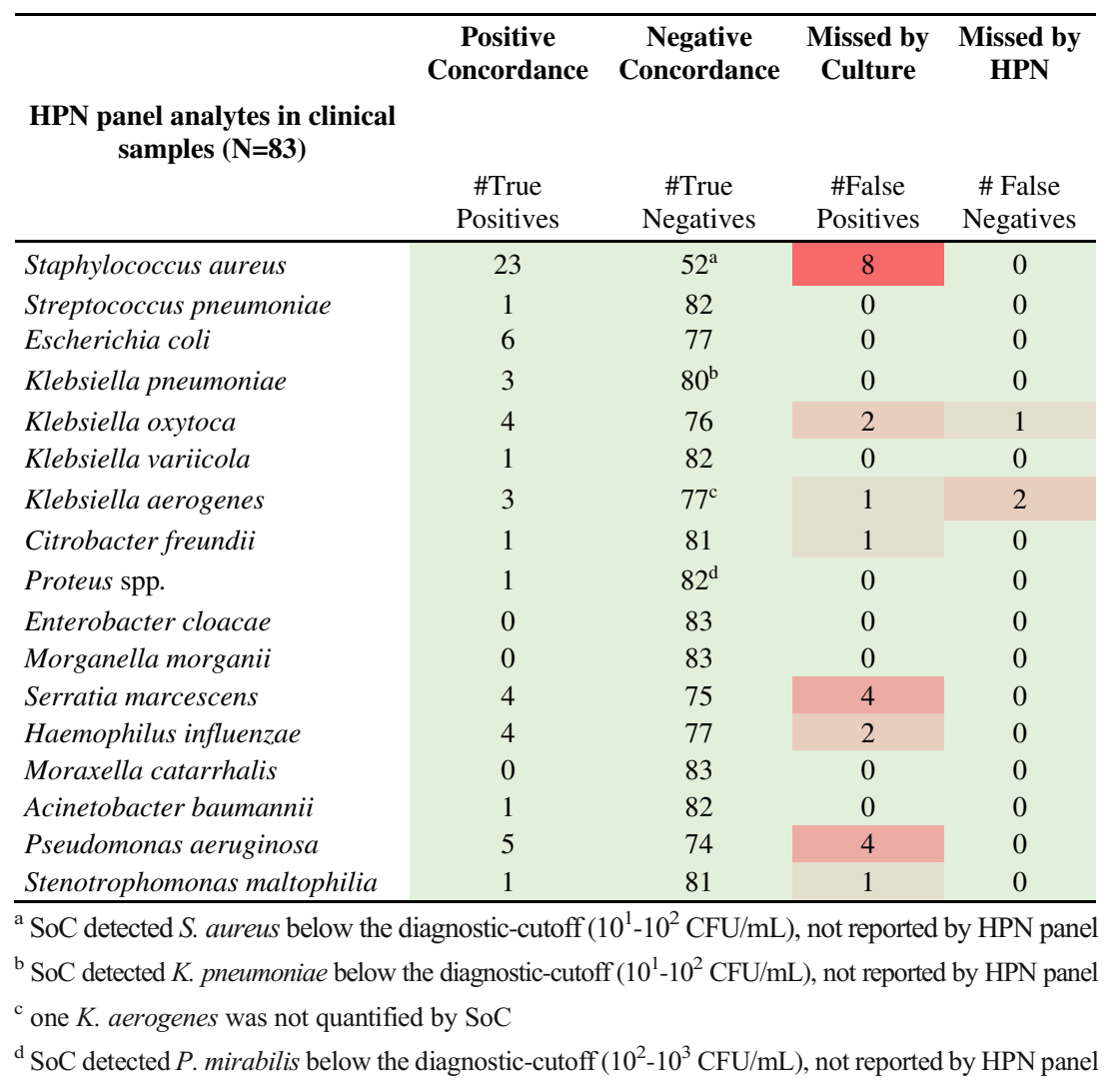


In total, the HPN Application could not detect an organism or had a discordant result in only $3 / 83$ $(3.6 \%)$ of the samples that were positive by culture for any of the panel organisms. Those three samples were (i) tracheal secretion sample that was blood-tinged, positive for $K$. aerogenes $>10^{5} \mathrm{CFU} / \mathrm{mL}$ by culture and HPN did not detect any organism, (ii) tracheal secretion sample positive for $K$. aerogenes $>10^{5} \mathrm{CFU} / \mathrm{mL}$ by culture and HPN detected $S$. aureus, and (iii) BAL sample positive for $S$. aureus $\left(10^{4} \mathrm{CFU} / \mathrm{mL}\right)$ and $K$. oxytoca $\left(10^{4} \mathrm{CFU} / \mathrm{mL}\right)$ by culture and the HPN Application detected only $S$. aureus. On the other hand, the HPN Application concorded fully with the culture results from $59(71.1 \%)$ samples (Table S3) and detected additional organisms among 21 (25.3\%) samples (Table S4). Of the 11 non-panel organisms found on SoC (Table S2), 55\% (6/11) were common oropharyngeal colonizing organisms that generally do not necessitate antibiotic escalation, and a further $45.5 \%$ (5/11) were organisms that would have been covered with empirical treatment.

\section{Discussion}

The need for early initiation of pathogen-specific antimicrobial therapy among patients with severe pneumonia using rapid diagnostic techniques (RDTs) was emphasized long before the current COVID-19 pandemic began. We report here the performance characteristics of the Unyvero HPN Application for the detection of bacterial species from native lower respiratory tract (LRT) samples, with predetermined microbiological culture results, obtained from critically ill COVID-19 patients. The Unyvero HPN Application detected additional bacterial species in $25.3 \%(21 / 83)$ samples and failed to detect a bacterial species in only $3.6 \%(3 / 83)$ samples tested in the

Table 3 Concordance between HPN Application and SoC for HPN panel organism

\begin{tabular}{ll}
\hline & No of samples (\%) \\
\hline Concordant results & $59(71.1)$ \\
SoC positive, HPN positive & $33(39.8)$ \\
SoC negative, HPN negative & $26(31.3)$ \\
Discordant results & $10(12.0)$ \\
HPN negative, SoC positive for panel organism & $1(1.2)$ \\
SoC negative, HPN positive & $8(9.6)$ \\
HPN and SoC identifying different organisms & $1(1.2)$ \\
Both concordant and discordant & $14(16.9)$ \\
SoC positive, HPN (additional organism) positive & $13(15.7)$ \\
HPN positive, SoC (additional organism) positive & $1(1.2)$ \\
\hline
\end{tabular}

Table 4 No. (\%) of HPN panel organism isolated from each sample compared to $\mathrm{SoC}$

\begin{tabular}{lllllll}
\hline & & & & & \\
\cline { 3 - 7 } & & None & One & Two & Three & Total \\
\hline HPN panel & None & $26(76.5)$ & $1(2.7)$ & 0 & 0 & 27 \\
& One & $7(20.6)$ & $26(70.3)$ & $1(8.3)$ & 0 & 34 \\
& Two & $1(2.9)$ & $10(27)$ & $8(66.6)$ & 0 & 19 \\
& Three & 0 & 0 & $3(25)$ & 0 & 3 \\
& Total & 34 & 37 & 12 & 0 & 83 \\
\hline
\end{tabular}

present study. Further, the new method demonstrated excellent negative predictive value $(99.8 \%)$ and generated results within $5 \mathrm{~h}$ from the time of loading the sample.

A recent study of the BioFire FilmArray Pneumonia panel concluded that the use of molecular diagnostic tools and the initiation of narrow-spectrum antibiotics are key elements of COVID-19 antimicrobial stewardship guidelines in critically ill [8]. Based on the recent estimates, nearly $80 \%$ of the hospitalized patients with COVID-19 are currently receiving antibiotics, albeit in the absence of a microbiological confirmation of the bacterial infection in large number of patients [2]. The rationale for antibiotic treatment in patients with COVID19 seems to be based on the prior experience with bacterial super-infections that were reported in nearly $11-35 \%$ patients with influenza viral infection [9]. Currently, it remains unclear whether bacterial co-infections are common among patients with SARS-CoV-2 infection, at the time of their admission to hospital; however, there is adequate evidence in the published literature suggesting that bacterial super-infections are common among COVID-19 patients admitted to the intensive-care units $[2,10,11]$. It is most likely possible that the bacterial super-infections among COVID-19 patients admitted to critical care units are due to the longer durations of stay in the ICU and mechanical ventilation, rather than the viral infection itself, but nonetheless, this requires diligent microbiological testing because the signs and symptoms can be similar and confounding. Given this context, the Unyvero HPN Application can be a potential RDT of choice, considering that the HPN panel is able to detect 20 bacterial species, one fungus, and 17 antimicrobial resistance genes (Table 1), that includes the most common infectious etiology of both healthcare- and ventilator-associated pneumonia.

In general, diagnostic yields from LRT samples vary with the sample type used. Invasive samples such as BAL and PSB are considered to have a better yield of the causative etiology of respiratory infections, as compared with non-invasive samples such as sputum. Diagnostic thresholds for various LRT samples among patients with health-care acquired pneumonia (HAP) range between $\geq 10^{3} \mathrm{CFU} / \mathrm{mL}$ for PSB samples to $\geq 10^{5}$ 
CFU/mL for aspirates and sputum [5]. A very low yield of pathogens from sputum samples of hospitalized patients with severe form of COVID-19 was recently reported [12]. Invasive sampling techniques have been contraindicated among COVID-19 patients outside the intensive-care units, due to the risk of aerosol generation. In the present study, tracheal aspirate was the predominant $(61,73.5 \%)$ sample type used. Nevertheless, we also included 19 (23\%) BAL or PSB samples among which four (one BAL and 3 PSB) samples had a bacterial species, isolated by culture at counts $\leq 10^{3}$ $\mathrm{CFU} / \mathrm{mL}$. The Unyvero HPN Application flagged all these four samples as negative (Table 2). Considering the lower diagnostic threshold, recommended for PSB samples in comparison with other sample types (aforementioned), diagnostic efficacy of the HPN Application for PSB samples in particular, needs further evaluation.

In our study, the HPN Application detected additional bacterial species among 21/83 (25.3\%) samples tested. This finding is in concordance with previous studies that have reported a similar increase in the bacterial yield from LRT samples, using other molecular detection assays among non-COVID19 patients [13-15]. Currently, the clinical implications of detecting additional bacterial species only by the molecular methods (in the absence of culture confirmation) remain unclear and most often it is speculated that the higher yield of the molecular tests can be attributed to their ability to detect nonviable bacteria from a past infection [15]. However, in our study, we also observed that the HPN Application could detect a bacterial pathogen from samples (from patients 4, 5, 9, and 11 in Table S1) that were negative by culture initially, but subsequent cultures ordered on these patients during the later course of their hospital stay were in fact positive for the same pathogen, indicating that the HPN panel can detect potential pathogens earlier than culture, which may enable earlier treatment and management of patients. Furthermore, the HPN Application demonstrated high negative predictive value of $99.8 \%$, which would allow for reduction in unnecessary antibiotic use and support antibiotic stewardship efforts. Given this context, perhaps prospective diagnostic trials in the near future may assess the true positive predictive values of the Unyvero HPN Application and other similar commercially available molecular RDTs.

Our study has a few limitations. Currently, we do not have the clinical data of the patients from whom the present study samples were obtained. Because of this we could not determine the (i) the proportion of samples that were sent to the microbiology laboratory prior the administration of antibiotics and (ii) the proportion of samples that were false positive by the HPN Application due to the detection of bacterial DNA from a past infection. Another limitation of the present study is that we could not determine the performance characteristics of the HPN Application for the detection of genes conferring antimicrobial resistance, due to the fact that only few samples yielded drug resistant phenotypes by culture in this study cohort (data not shown here). Despite these limitations, our study identified that the Unyvero HPN Application is a reliable and rapid diagnostic test with excellent negative predictive value for detection of bacterial pathogens from lower respiratory tract samples.

In conclusion, rapid diagnostics such as the Unyvero HPN panel are imperative to evaluate and test patients for bacterial pneumonia earlier in their hospital journey for more prompt and appropriate treatment. Current diagnostic tools for hospital-acquired pneumonia are limited; the Unyvero multiplex PCR panel provides benefit for these patients by enabling rapid diagnosis of pathogens of concern. Unyvero HPN demonstrated a higher diagnostic yield than culture; it is significantly faster, with turnaround time of $<5 \mathrm{~h}$ from sample to results compared with average of 2.5 days for culture, providing clinicians earlier data to inform antimicrobial decisions, especially in critically ill COVID-19 patients and the upcoming flu season.

Supplementary Information The online version contains supplementary material available at https://doi.org/10.1007/s10096-021-04194-6.

Acknowledgements The authors thank Curetis, Germany GmbH, for kindly providing the HPN cartridges for the present evaluation. Preliminary results were submitted as an abstract at the ESCMID Conference on Coronavirus Disease (ECCVID), September 2020.

Authors' contributions Both authors CT and CGG contributed to the study conception and design. The first draft of the manuscript was written by $\mathrm{CT}$, and CGG reviewed and approved the final version of the manuscript.

Funding Open access funding provided by Karolinska Institute.

Data availability All relevant data are available in the manuscript and the supplementary files.

\section{Declarations}

Consent to participate Not applicable.

Consent to publish Not applicable.

Ethics approval Ethical permit was obtained from the Swedish Ethics Review Authority (Ref No:2020-04999).

Conflict of interest The authors declare that they have no conflict of interest.

Open Access This article is licensed under a Creative Commons Attribution 4.0 International License, which permits use, sharing, adaptation, distribution and reproduction in any medium or format, as long as you give appropriate credit to the original author(s) and the source, provide a link to the Creative Commons licence, and indicate if changes were made. The images or other third party material in this article are included in the article's Creative Commons licence, unless indicated 
otherwise in a credit line to the material. If material is not included in the article's Creative Commons licence and your intended use is not permitted by statutory regulation or exceeds the permitted use, you will need to obtain permission directly from the copyright holder. To view a copy of this licence, visit http://creativecommons.org/licenses/by/4.0/.

\section{References}

1. Garau J, Baquero F, Perez-Trallero E, Pérez J-L, Martin-Sanchez A, Garcia-Rey C, Martin-Herrero J, Dal-Ré R (2008) Factors impacting on length of stay and mortality of community-acquired pneumonia. Clin Microbiol Infect 14(4):322-329

2. Langford BJ, So M, Raybardhan S, Leung V, Westwood D, MacFadden DR, Soucy JR, Daneman N (2020) Bacterial coinfection and secondary infection in patients with COVID-19: a living rapid review and meta-analysis. Clin Microbiol Infect 26(12):1622-1629

3. Lansbury L, Lim B, Baskaran V, Lim WS (2020) Co-infections in people with COVID-19: a systematic review and meta-analysis. J Infect 81(2):266-275

4. Cox MJ, Loman N, Bogaert D, O'grady J (2020) Co-infections: potentially lethal and unexplored in COVID-19. Lancet Microbe 1(1):e11

5. Kalil A, Metersky M, Klompas M, Management of adults with HAP/VAP•CID (2016) 63 (1 September)・ e61. Clin Infect Dis 2016:63

6. Kais M, Spindler C, Kalin M, Örtqvist Å, Giske CG (2006) Quantitative detection of Streptococcus pneumoniae, Haemophilus influenzae, and Moraxella catarrhalis in lower respiratory tract samples by real-time PCR. Diagn Microbiol Infect Dis 55(3):169-178

7. Wilson EB (1927) Probable inference, the law of succession, and statistical inference. J Am Stat Assoc 22(158):209-212

8. Verroken A, Scohy A, Gérard L, Wittebole X, Collienne C, Laterre P-F (2020) Co-infections in COVID-19 critically ill and antibiotic management: a prospective cohort analysis. Crit Care 24(1):1-3

9. Klein EY, Monteforte B, Gupta A, Jiang W, May L, Hsieh YH, Dugas A (2016) The frequency of influenza and bacterial coinfection: a systematic review and meta-analysis. Influenza Other Respir Viruses 10(5):394-403

10. Zhou F, Yu T, Du R, Fan G, Liu Y, Liu Z, Xiang J, Wang Y, Song B, Gu X, Guan L, Wei Y, Li H, Wu X, Xu J, Tu S, Zhang Y, Chen H, Cao B (2020) Clinical course and risk factors for mortality of adult inpatients with COVID-19 in Wuhan, China: a retrospective cohort study. Lancet 395(10229):1054-1062

11. Contou D, Claudinon A, Pajot O, Micaëlo M, Longuet Flandre P, Dubert M, Cally R, Logre E, Fraissé M, Mentec H, Plantefève G (2020) Bacterial and viral co-infections in patients with severe SARS-CoV-2 pneumonia admitted to a French ICU. Ann Intensive Care 10(1):119

12. Hughes S, Troise O, Donaldson H, Mughal N, Moore LS (2020) Bacterial and fungal coinfection among hospitalized patients with COVID-19: a retrospective cohort study in a UK secondary-care setting. Clin Microbiol Infect 26(10):1395-1399

13. Webber DM, Wallace MA, Burnham CA, Anderson NW (2020) Evaluation of the BioFire FilmArray pneumonia panel for detection of viral and bacterial pathogens in lower respiratory tract specimens in the setting of a tertiary care academic medical center. J Clin Microbiol 58(7):e00343-20

14. Gadsby NJ, McHugh MP, Forbes C, MacKenzie L, Hamilton SK, Griffith DM, Templeton KE (2019) Comparison of Unyvero P55 Pneumonia Cartridge, in-house PCR and culture for the identification of respiratory pathogens and antibiotic resistance in bronchoalveolar lavage fluids in the critical care setting. Eur J Clin Microbiol Infect Dis 38(6):1171-1178

15. Murphy CN, Fowler R, Balada-Llasat JM, Carroll A, Stone H, Akerele O, Buchan B, Windham S, Hopp A, Ronen S, Relich RF, Buckner R, Warren DA, Humphries R, Campeau S, Huse H, Chandrasekaran S, Leber A, Everhart K, Harrington A, Kwong C, Bonwit A, Dien Bard J, Naccache S, Zimmerman C, Jones B, Rindlisbacher C, Buccambuso M, Clark A, Rogatcheva M, Graue C, Bourzac KM (2020) Multicenter evaluation of the BioFire FilmArray pneumonia/pneumonia plus panel for detection and quantification of agents of lower respiratory tract infection. J Clin Microbiol 58(7):e00128-20

Publisher's note Springer Nature remains neutral with regard to jurisdictional claims in published maps and institutional affiliations. 